\title{
Acquired equivalence of cues in pigeon autoshaping: Effects of training with common consequences and with common antecedents
}

\author{
CHARLOTTE BONARDI, VEREMUNDO REY, MARK RICHMOND, \\ and GEOFFREY HALL \\ University of York, York, England
}

\begin{abstract}
In three experiments, we sought evidence for the acquired equivalence of cues in pigeons trained in an autoshaping paradigm. In Experiment 1, presentations of each of a pair of cues (different keylight stimuli) preceded a common consequence (a different keylight stimulus). The pattern of response then established by further training given to one member of the pair was found to generalize preferentially to the other, demonstrating equivalence between cues that had shared a common consequence. The same test procedure was used in Experiment 2, but with a training procedure in which each cue of a pair was preceded by a given stimulus. This too resulted in enhanced generalization between members of the pair, showing that equivalence can be established when cues have been experienced along with a common antecedent. Both training procedures were combined in Experiment 3 to confirm the reliability of the effects previously obtained. The discussion is focused on ways in which the associative explanation offered for cases of equivalence mediated by a common consequence might be extended to accommodate equivalence mediated by a common antecedent.
\end{abstract}

Two stimuli may be said to be equivalent in some respect when one can function as a substitute for the other in that respect. Primary stimulus generalization provides an example: a test stimulus may be said to be equivalent to a conditioned stimulus (CS) to the extent that it evokes the conditioned response (CR) that occurs to the CS. Such equivalence is usually explained in terms of the assumption that the stimuli in question share a number of features or elements. It has also been suggested, however, (see Hall, 1991, for a review) that certain forms of training might render two stimuli equivalent (and thus allow generalization between them) even when the stimuli share no, or very few, elements. In particular, it has been suggested that cues will acquire equivalence when they are subjected to training that allows each to become linked to a common consequence.

Perhaps the earliest clear statement of the notion of acquired equivalence was made by Miller and Dollard (1941; see also Miller, 1948), who suggested that, for humans, two cues might become equivalent when the subject was trained to respond to each with the same verbal response. This suggestion prompted a large body of ex-

This work was supported by grants from the Science and Engineering Research Council and from the York University Research Priming Fund. We thank R. C. Honey for much helpful discussion. V.R. is now at the University of Oviedo, Spain. Correspondence concerning this article should be addressed to G. Hall, Department of Psychology, University of York, York, YO1 5DD, U.K. perimental work (reviewed by Hall, 1991) that tended on the whole to provide confirmation. More recent work has extended the range of the phenomenon by demonstrating its occurrence in nonhuman subjects and in procedures that involve Pavlovian rather than instrumental training (and in which, therefore, the common consequence is taken to be a stimulus rather than a response). Honey and Hall (1989), for example, found that an initial phase of Pavlovian training, in which each of two auditory cues signaled the delivery of a food pellet for rats, produced an increase in the likelihood that a different CR (conditioned suppression) subsequently acquired by one of the cues would generalize to the other.

The explanation offered by Honey and Hall (1989) for their results was in essence the same as that proposed by Miller and Dollard (1941), which was itself based on Hull's (1939) notion of secondary or mediated generalization. According to Hull (1939), attaching a common response to two different stimuli means that each stimulus evokes the same set of response-produced cues. These cues would form part of the complex evoked by subsequent presentations of either of the stimuli and would form a basis on which some new behavior conditioned to one of them might generalize to be evoked by the other. Applying this type of analysis to their Pavlovian procedure, Honey and Hall (1989) argued that the initial phase of appetitive training would allow the CS to activate a representation of food during subsequent conditioned suppression training. This associatively activated represen- 
tation could itself then become associated with the aversive unconditioned stimulus (US). The test stimulus, because it too will activate the food representation, would thus also be rendered capable of activating the US representation and thus of evoking, to some extent, the CR of conditioned suppression. The food representation can be viewed as constituting an extra common element added to each of the auditory cues by associative learning.

Our purpose in performing the experiments reported here was to confirm the generality of the basic acquired equivalence effect and to extend its range to procedures that would also allow us to evaluate the associative interpretation. In Experiment 1, we attempted to devise an analogue of Honey and Hall's (1989) procedure, by using pigeons trained in an autoshaping procedure. The event used as a common consequence during the phase of training designed to establish equivalence was one lacking primary motivational significance (being the illumination of a response key rather than the presentation of food employed by Honey \& Hall, 1989). The target CR trained in the second phase (and used to assess the degree of generalization between the supposedly equivalent stimuli) was food-reinforced keypecking (rather than the conditioned suppression used by Honey \& Hall, 1989).

In Experiments 2 and 3, we used the same basic training procedure but investigated the possibility that equivalence between stimuli might also be established by training in which they shared not a common consequence but a common antecedent. Studies of acquired equivalence in nonhuman subjects have concentrated almost without exception (but see Urcuioli \& Zentall, in press) on the former procedure, and our associative account has been designed to deal with its effects. It remains possible, however, that acquired equivalence is a more general phenomenon than has hitherto been supposed-that treating two stimuli in the same way may render them equivalent whether this treatment associates them with a common consequence or not. A range of possibilities deserves investigation, but the "backward" procedure (in which the target stimuli are given a common antecedent) seems the most obvious starting point, in part because a demonstration of acquired equivalence in this case would constitute an interesting challenge to the associative account offered for the standard "forward" case.

\section{EXPERIMENT 1}

The design of this experiment is summarized in Table 1. In the first stage, subjects received training designed to establish equivalence between pairs of keylight stimuli by giving each member of the pair a common consequence. To this end, a serial conditioning procedure was used. Stimuli $A$ and $C$ were both followed on each presentation by stimulus $X$; stimuli $B$ and $D$ were followed by $Y$. Food was presented at the offset of the second element on each trial, to ensure that attention to the keylight stimuli would be maintained. In Stage 2, training was given to just one member of each pair. Presentations of $C$ were followed
Table 1

Experimental Designs

\begin{tabular}{ccc}
\hline Stage 1 & Stage 2 & Test \\
& Experiment 1 & \\
$A \rightarrow X+$ & $C+$ & $A \& B$ \\
$C \rightarrow X+$ & $D-$ & \\
$B \rightarrow Y+$ & & \\
$D \rightarrow Y+$ & Experiment 2 & \\
& & $A \& B$ \\
$X \rightarrow A+$ & $C+$ & \\
$X-C+$ & $D-$ & \\
$Y \rightarrow B+$ & & \\
$Y \rightarrow D+$ & & \\
\hline
\end{tabular}

Note- $A, B, C, D, X$, and $Y$ represent different keylight stimuli; " +" denotes the presentation of food and " -" its absence. In Stage 1, two stimuli were presented sequentially prior to food. All animals experienced all trial types in each stage.

by immediate food reinforcement, and presentations of $D$, by no other event. It was anticipated that a high rate of autoshaped responsing to $C$ would develop, whereas the tendency to respond to $D$ would decline from the level established during Stage 1. The question of interest was how the response tendencies established in Stage 2 would generalize to the test stimuli in the final (test) stage. If Stage 1 training engendered equivalence, it might be expected that the high rate of response to $C$ would generalize more readily to $A$ than to $B$; on the other hand, the tendency to respond at a low rate to $D$ would generalize more to $B$ than to $A$. The anticipated test result, therefore, was that $A$ would evoke a higher level of responding than $B$, in spite of the fact that these two stimuli did not themselves receive differential treatment in the earlier stages of training.

\section{Method}

Subjects and Apparatus. The subjects were 16 locally obtained pigeons maintained at $80 \%$ of their free-feeding body weights (mean, $513 \mathrm{~g}$; range, 388-638 g). They were housed in individual cages in a colony room that was lit from 800 to $2400 \mathrm{~h}$ each day. They had served previously as subjects in an experiment in which the autoshaping procedure was used, but they had not received exposure to the keylight stimuli that were to be used in this experiment.

Training was conducted in four standard three-key pigeon chambers supplied by Campden Instruments Ltd. Only the center key was used. An in-line projector behind the key allowed the following stimuli to be presented: yellow, purple, horizontal striations, vertical striations, a dot, and a polygon. The colors were presented over the entire key (a $2.5-\mathrm{cm}$-diam circle). The other stimuli consisted of white figures against a dark background presented in the center of the key. The striations consisted of three parallel white stripes, $2 \mathrm{~mm}$ wide, $2 \mathrm{~mm}$ apart, and $1 \mathrm{~cm}$ long; the dot was a $1-\mathrm{cm}$ diam circle; the polygon was produced by superimposing two $1-\mathrm{cm}$ equilateral triangles, one erect and one inverted, with the apex of each touching the base of the other. A grain feeder positioned just above floor level and immediately below the center key was used to provide reinforcement. The chamber was illuminated by a $2.8-\mathrm{W}$ bulb, rated for $24 \mathrm{~V}$ but operated at $15 \mathrm{~V}$. Each chamber was enclosed in a sound-attenuating shell. The operation of an extractor fan provided background masking noise.

Procedure. The birds' previous experience of autoshaping meant that all would eat readily from the feeder and peck at illuminated 
response keys. Stage 1 consisted of 12 training sessions of 24 trials, 6 each of the four trial types shown in Table 1. Sessions were given twice daily. Each trial began with the presentation of a keylight stimulus $(A, B, C$, or $D)$ for $10 \mathrm{sec}$. The offset of this stimulus was followed immediately by a 10 -sec presentation of either $X$ or $Y$, which was itself followed by a 5-sec operation of the feeder. The interval between the end of one trial and the beginning of the next varied between 75 and $115 \mathrm{sec}$, with a mean of $95 \mathrm{sec}$. For half the subjects, stimulus $A$ consisted of horizontal striations, and $B$ of vertical striations; for the other half, the arrangement was reversed. Half of each of these groups received the dot as stimulus $C$ and the polygon as $D$, and the other half received the reverse. Finally, for half the subjects in each of these subgroups, stimulus $X$ was purple and $Y$ was yellow, and for half, the assignment was reversed. Trials were ordered as three blocks of 8 , each block containing 2 of each trial type. Within a block, the order of presentation was random.

The eight sessions of Stage 2 each comprised 24 trials, 12 with stimulus $C$ and 12 with $D$, order of presentation being determined by a random sequence. The offset of $C$ was followed by food; $D$ was not reinforced. The test stage consisted of a single session containing 24 nonreinforced trials, 12 with $A$ and 12 with $B$. These were organized as three 8-trial blocks. For half the subjects, the sequence of trials in each block was $A B B A B A A B$; for the remaining subjects, the positions of $A$ and $B$ were interchanged. For each subject, a separate record was kept of the responding elicited by the first presentation of each of the test stimuli. As before, stimulus duration was $10 \mathrm{sec}$ and the mean interval between trials was $95 \mathrm{sec}$.

\section{Results}

By the end of Stage 1 training, the subjects were responding readily to $X$ and $Y$, the stimuli that immediately preceded reinforcement, and at a rather lower rate to stimuli $A, B, C$, and $D$ (see Figure 1). The rates governed by $X$ and $Y$ in the last session of Stage 1 (25.75 and 21.03 responses $/ \mathrm{min})$ did not differ significantly $(N=16$, Wilcoxon $T=38$ ). The rates of responding to $C$ and $D$, the stimuli that were to undergo discrimination training in Stage 2, did not differ $(N=12, T=27.5)$, nor did the rates of responding to $A$ and $B$, the pair of stimuli to be used in the test phase $(N=15, T=55)$. Discrimination training in Stage 2 established a difference in the responding controlled by stimuli $C$ and $D$. The rate of responding to $D$ (already low at the end of Stage 1 ) declined

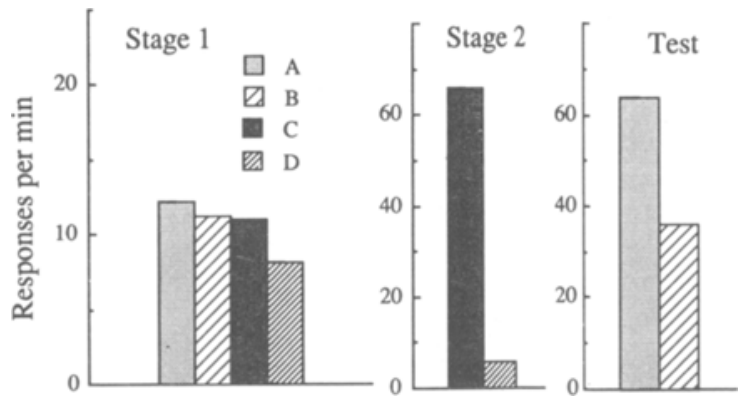

Figure 1. Group mean performance in Experiment 1. A, B, C, and $D$ represent four different keylight stimuli. Scores for Stages 1 and 2 are for the final session of each stage; test scores are for the first test trial of each type. slightly; that to $C$ increased markedly. By the end of this phase, rates of responses to these two stimuli (see Figure 1) differed reliably $(N=15, T=3, p<.01)$.

Response rates pooled over the entire test session were similar to those recorded for the end of Stage 1 and did not differ significantly (for $A$, the rate was 11.22 responses/min and for $B, 11.19 ; N=14, T=49.5$ ). But this session was carried out in extinction, and the pooled results obscure the fact that the response rates were much higher on the early trials, on which the rates of response to $A$ and $B$ also differed substantially. As Figure 1 shows, stimulus $A$ evoked much more responding on its first presentation than did stimulus $B(N=12, T=6.5$, $p<.01$ ). There was some tendency on the remaining trials of the session (11 with stimulus $A$ and 11 with $B$ ) for responding to $B$ to be higher than that to $A$, but rates were low and the difference was not statistically reliable (6.61 responses/min to $A$ vs. 8.93 to $B ; N=14, T=23$ ).

\section{Discussion}

Although they had not themselves been associated with different schedules of reinforcement, stimuli $A$ and $B$ were found to control different levels of responding on test. The rate of responses to $A$ was high, like that to the reinforced stimulus $C$; the rate to $B$ was relatively low, like that to the nonreinforced stimulus $D$. This equivalence between stimulus pairs was a consequence of the initial phase of training in which $A$ and $C$ had been associated with one consequence $(X)$, and $B$ and $D$, with another $(Y)$

This result extends the range of procedures in which acquired equivalence has been demonstrated, but it is formally analogous to those used previously and can be explained in terms of the associative mechanism discussed by Honey and Hall (1989; see also Honey \& Hall, 1991). Consider the stimulus pair $A$ and $C$ (a parallel argument applies to the pair $B$ and $D$ ). In Stage 1 training, it may be assumed that each of these stimuli forms an association with the representation of the event $X$ that follows them. This will allow the presentation of $C$ during Stage 2 to activate the $X$ representation, which could then itself become associated with the immediate reinforcement that occurs during this stage. Finally, in the test stage, Stimulus $A$, although it has not itself been directly reinforced, will be able to evoke a relatively high response rate by virtue of its ability to activate the $X$ representation.

In its design and results, the experiment reported here constitutes an interesting parallel to those on the "common coding" effect studied by Urcuioli, Zentall, JacksonSmith, and Steirn (1989; see also Zentall, Steirn, Sherburne, \& Urcuioli, 1991) in pigeon conditional discrimination learning ("symbolic matching to sample"). Although different in its procedural details, their basic experiment can be interpreted as being formally identical to that just described. In a first stage of training, the two critical stimuli (we may again call them $A$ and $C$ ) were given a common outcome-in this case, each was used as a conditional cue (or sample) indicating that the 
choice of comparison stimulus $X$ rather than $Y$ would be rewarded. In a second phase, $C$ acquired a new property, being trained as the sample for a quite different conditional discrimination. The final phase showed that, without further training, stimulus $A$ was able to function adequately as the sample stimulus for choice between the comparison stimuli trained with $C$ in the second phase; that is, in the terminology used here, $A$ and $C$ had acquired equivalence.

It may be possible to extend our associative analysis to deal with the effects demonstrated in conditional discrimination learning. It is necessary to assume that the sample stimulus in a conditional discrimination can become associatively linked to the rewarded comparison stimulus that follows it, an assumption that may be justified by the fact that these two events will regularly be experienced in close succession once the task has begun to be learned. In the Urcuioli et al. (1989) experiment, as described above, sample stimuli $A$ and $C$ would both become linked with the comparison stimulus $X$ in the first phase of training. These links would then be able to mediate generalization between $A$ and $C$, allowing $A$ to serve as substitute for $C$ in the new task trained in the second stage. Whether this associative mechanism is indeed the basis of the common-coding effect or of our own acquired equivalence effect, for that matter, remains to be determined. We hoped that our next experiment might produce results bearing on this issue.

\section{EXPERIMENT 2}

In this experiment, we investigated the possibility that stimuli might also be rendered equivalent by training in which they were experienced as having the same antecedent. The design of the experiment, outlined in Table 1, follows that of Experiment 1, differing only in that the order of stimulus presentation in Stage 1 was reversed. Thus, the pairs of stimuli between which it was hoped equivalence might be established were preceded by a common event: $A$ and $C$ by stimulus $X, B$ and $D$ by stimulus $Y$. Stage 2 was again designed to enhance the response rate to $C$ and to reduce it to $D$, and the test phase looked for equivalent changes in responding to $A$ and $B$.

Our interpretation of the equivalence effect found in Experiment 1 relied on the notion that associations formed during the first stage of training would allow each of the members of the pair of critical stimuli ( $A$ and $C$, say) to activate the representation of a third event $(X)$. Now, in that experiment, $A$ and $X$ were presented in a standard forward conditioning arrangement, as were $C$ and $X$. In the procedure to be used here, however, the arrangement was reversed, making it impossible (according to associative theories that allow only "forward" or anticipatory associations) for the animals to acquire the associations necessary for equivalence to be mediated by the associatively activated representation of the $X$ stimulus. A demonstration of equivalence in this backward training procedure would make it necessary for us to reconsider or to extend the associative explanation that had served thus far.

\section{Method}

The subjects were a further 16 pigeons (mean free-feeding body weight, $366 \mathrm{~g}$; range, 285-500 g), maintained and housed as in Experiment 1 . They had served previously in an experiment performed with the autoshaping procedure and had experienced some of the geometrical shapes used as stimuli in Experiment 1. Accordingly, the shapes used in that experiment were replaced by the following: a $1-\mathrm{cm}$ square, an inverted $1-\mathrm{cm}$ equilateral triangle, a grid produced by superimposing the horizontal and vertical striations used in Experiment 1, a diamond (the $1-\mathrm{cm}$ square rotated $45^{\circ}$ ), and an eight-pointed star produced by presenting the square and the diamond simultaneously.

In Stage 1, all subjects received the four trial types shown in Table 1 . Stimuli $X$ and $Y$ were the purple and yellow keylights; $C$ and $\mathrm{D}$ were the star and the grid. For half the subjects, stimulus $A$ was the triangle and $B$ the diamond; for the other half, the reverse arrangement was employed. In Stage 2, all subjects received discrimination training with the star reinforced and the grid nonreinforced. The test consisted of nonreinforced presentations of $A$ and $B$ with the trials scheduled as in Experiment 1. As before, it was intended to record results separately for the first presentation of each of the test stimuli but an error led to the loss of these data. Fortunately, however, the effect evident on test turned out to be more long-lived than that of Experiment 1, and it proved worthwhile to conduct a total of four test sessions. In respects not specified here, the procedure was the same as that described for Experiment 1.

\section{Results and Discussion}

Figure 2 shows the mean response rates controlled by stimuli $A, B, C$, and $D$ on the last session of Stage 1 training. Since, in this experiment, these stimuli had immediately preceded reinforcement in Stage 1 , the rates were somewhat higher than those observed in Experiment 1. All evoked approximately the same rate of response at this stage; in particular, there was no significant difference between $C$ and $D$, the pair later subjected to discrimination training in Stage $2(N=14, T=30)$, nor between $A$ and $B$, the stimuli presented in the test $(N=13$, $T=43$ ). Rates to $X$ and $Y$, which in this experiment were presented as the first elements of serial compounds, were low (8.97 responses/min to $X$ and 6.25 responses/min to $Y)$ and did not differ significantly $(N=13, T=22)$.
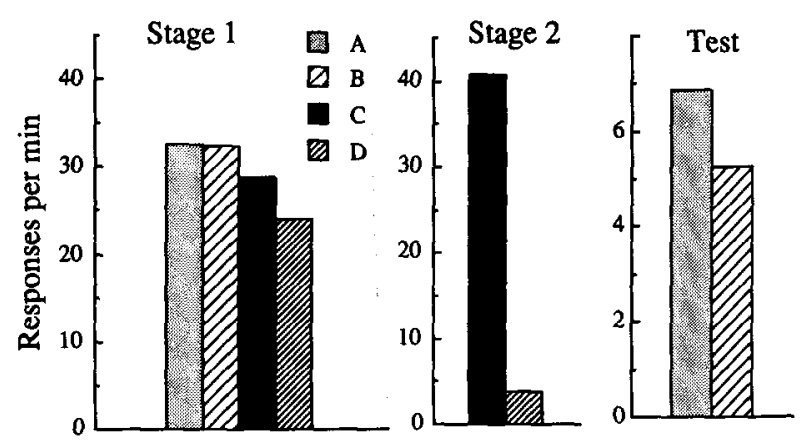

Figure 2. Group mean performance in Experiment 2. A, B, C, and $D$ represent four different keylight stimuli. Scores for Stages 1 and 2 are for the final session of each stage; test scores are pooled over all test sessions. 
Stage 2 training produced an increase in response rate to stimulus $C$ and a reduction to stimulus $D$ so that, by the end of this stage (see Figure 2), the rates elicited by these two stimuli differed significantly $(N=15, T=0$, $p<.01$ ). This difference between $C$ and $D$ was reflected in the performance shown to $A$ and $B$ in the test. Although the overall rates shown in the figure are low (the test was carried out in extinction, and the scores given are averaged over the test session), some responding occurred during all four test sessions. In each session, the rate of responding to $A$ was higher than that to $B$. Statistical analysis conducted on responding totalled over all test sessions (see Figure 2) showed the difference to be reliable $(N=16$, $T=26, p<.05$ ). We conclude, therefore, that equivalence had been established between $A$ and $C$ and between $B$ and $D$ by virtue of the Stage 1 training in which each member of a given pair was experienced following a common antecedent.

After this experiment had been completed, we became aware of a study by Urcuioli and Zentall (in press) in which they attempted to show, using their conditional discrimination task, that comparison stimuli might become functionally equivalent as a result of training in which they had been preceded by the same sample stimulus (what they refer to as "one-to-many" training). Just as the "manyto-one" procedure of Urcuioli et al. (1989) parallels the design of our Experiment 1, so does this one-to-many training procedure parallel Experiment 2 . In essence, the design used by Urcuioli and Zentall (in press) was as follows (cf. Experiment 2, Table 1). In a first stage of training, birds learned to choose comparison stimulus $A$ when it was presented after sample $X$, and also, on other trials, to choose $C$ after $X$. They then learned a new conditional discrimination in which a novel sample stimulus was used to indicate that $C$ should be chosen. The test phase asked whether this new sample would control a tendency to choose $A$ when it was again presented as a comparison stimulus. There were marked individual differences in test performance, and only 2 of the 12 subjects studied by Urcuioli and Zentall (in press) showed any real transfer from the earlier phases of training. These 2 , however, showed a substantial preference for the $A$ comparison stimulus, a finding that, taken together with the present results, supports the conclusion that the experience of two events as having a common antecedent can establish equivalence between them.

\section{EXPERIMENT 3}

The aims of this experiment were to confirm the reliability of the effects observed in Experiments 1 and 2, and, by including the forward and backward training procedures in one experiment, to allow the possibility of a direct comparison between them. In addition, we introduced a procedural change in the hope of enhancing the magnitude of the effects obtained. (The difference in test performance in responses to stimuli $A$ and $B$ was shortlived in Experiment 1 and, although more sustained, was of no great size in Experiment 2.) In our previous experiments, Stage 1 training was reinforced, with food following each presentation of a serial compound (see Table 1). There was reason to think that this procedure might act to diminish the size of the effect that we were looking for in the test phase. Honey and Hall (1989), in their study of acquired equivalence in rats, demonstrated that equivalence could be established between a pair of cues by training in which both were experienced prior to food. If the mechanism at work in that experiment was operating here, our present Stage 1 training procedure might be expected to establish a degree of equivalence between all the keylight stimuli presented in that stage. Equivalence produced by the common relationship between the keylights and food would act to obscure the differential generalization that we hoped to observe between $A$ and $C$ and between $B$ and $D$. Accordingly, Stage 1 training was nonreinforced in the present experiment. In an attempt to ensure that attention to the key was maintained during this phase, occasional reinforced trials with a white key were also presented.

\section{Method}

The subjects were 16 experimentally naive pigeons (mean freefeeding body weight, $432 \mathrm{~g}$; range, 319-556 g) maintained as in the previous experiments. The apparatus and keylight stimuli were the same as those in Experiment 1, apart from the addition of a plain white keylight stimulus and the replacement of the dot (which, it was felt, the birds might find difficult to discriminate from the white key) by a diamond (white, $1 \mathrm{~cm}$ per side, on a black background).

After magazine training, the subjects received a preliminary phase of autoshaping to establish responding to the white center key. All received a minimum of five daily 40 -min sessions during which trials occurred, on the average, every $60 \mathrm{sec}$. A trial consisted of the illumination of the white center key for $10 \mathrm{sec}$, followed by a 5-sec presentation of food. This was sufficient to establish responding in most birds, but those having low rates were given extra sessions as was necessary. On the day before the start of Stage 1, all birds were given a further 40 -min session of autoshaping with the intertrial interval increased to a mean of $120 \mathrm{sec}$. The birds were then assigned at random to one of two equal-sized groups for Stage 1.

There were 12 sessions of Stage 1, each comprising 25 trials. Group Forward received, in each session, five nonreinforced presentations of each of the following trial types: $A \rightarrow X, C \rightarrow X, B \rightarrow Y$, $D \rightarrow Y$. In addition, there were five reinforced presentations of the white key. The order of presentation of these various trial types was determined by a random sequence. The subjects in Group Backward were treated identically, apart from receiving $X \rightarrow A, X \rightarrow C$, $Y \rightarrow B$, and $Y \rightarrow D$ as the nonreinforced trials. There followed 10 sessions of Stage 2 training in which $C$ was followed by reinforcement and $D$ was not. The single test session consisted of 24 nonreinforced presentations of $A$ and $B$, organized as three blocks of trials occurring in the following sequence: $A B B A B A A B$. A separate record was made of total response to $A$ and $B$ after the first of these blocks. Any procedural details not specified here were the same as those described for the previous experiments.

\section{Results and Discussion}

Stage 1 training proceeded uneventfully. Responding was maintained to the white key but occurred only infrequently to the other stimuli. On the final session of this 
stage, only 3 of the 16 birds emitted responses to stimuli other than white. (Two of these were in Group Backward, the other in Group Forward. The bird with the highest level of responding made a total of only 28 responses in the session.) Group Forward had a mean rate of 46.20 responses/min and Group Backward of 78.30 on whitekey trials in this session. Although sizeable, this difference was not statistically reliable (Mann-Whitney $U=18, N_{1}=N_{2}=8$ ). The difference was probably not a consequence of Stage 1 training itself but a chance product of the initial assignment of animals to groups. Inspection of performance on the last day of preliminary autoshaping revealed a difference (again, large but not statistically reliable) between the rates for those subsequently assigned to Group Backward and those subsequently assigned to Group Forward (means of 61.80 and 33.34 responses/min, respectively; $U=20.5, N_{1}=$ $\mathrm{N}_{2}=8$ ).

Stage 2 discrimination training was successful in establishing, in both groups, a much higher rate of responses to stimulus $C$ than to stimulus $D$. In the final session of this stage, the rates for Group Backward were 128.13 responses/min to $C$ and 20.75 to $D(N=8, T=0, p<$ $.01)$. The equivalent scores for Group Forward were 76.69 to $C$ and 13.25 to $D(N=8, T=1, p<.05)$.

Figure 3 shows, separately for each group, test performance in response to stimuli $A$ and $B$. The lower part of the figure shows data pooled over the entire test session; the upper part of the figure shows the results for the first block of test trials. Rates were higher on the first
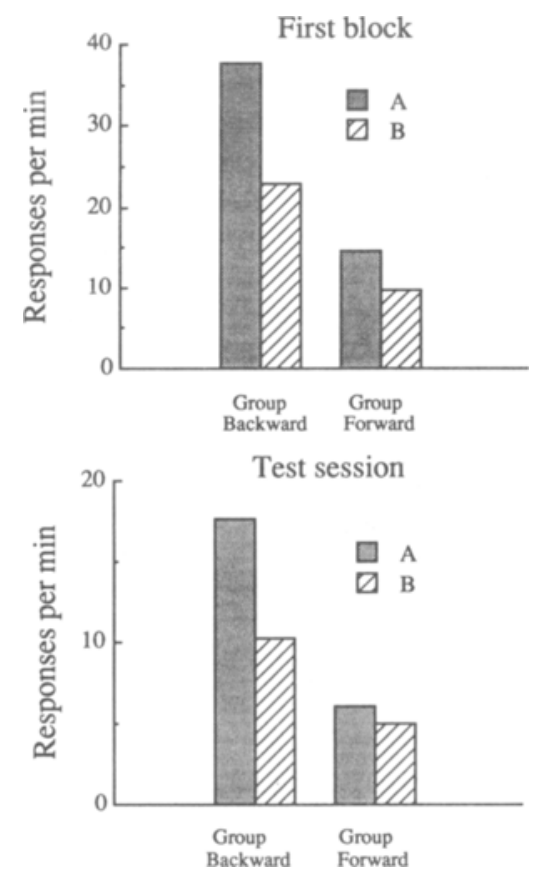

Figure 3. Group mean performance in the test session for the two groups of Experiment 3. The top panel shows results for the first block of test trials; the lower panel, for the entire test session. block than overall (the test was carried out in extinction), but both response measures show the same general features. First, the higher response rate evident in Group Backward from the start of training was present also in this test; second, stimulus $A$ tended to elicit more responding than did stimulus $B$, thus replicating the results of our previous experiments. Finally, there is some sign that the difference between $A$ and $B$ is more marked in Group Backward than in Group Forward. To assess the reliability of this apparent interaction, the data depicted in the figure were subjected to a factorial analysis of variance, the factors being group (forward or backward) and test stimulus $(A$ or $B)$.

The analysis conducted on the data recorded for the first block revealed no overall effect of group $[F(1,14)=$ 2.12], but a significant difference between the rates governed by the $A$ and $B$ stimuli $[F(1,14)=5.29, p<$ $.05]$. The interaction between the factors was not significant $[F(1,14)=1.35]$. Although not strictly legitimate given this nonsignificant interaction, the pattern of results shown in the figure encouraged us to carry out a simple effects analysis. This showed that the difference in rates of responding to $A$ and $B$ was significant for Group Backward $[F(1,14)=5.99, p<.05]$, but not for Group Forward $(F<1)$.

An analysis of variance conducted on the data for the entire session (lower panel of Figure 3) revealed no overall effect of group $[F(1,14)=1.77]$, but a near-significant difference in performance in response to $A$ and $B[F(1,14)$ $=3.55, p<.1]$. Again, the interaction between the factors was not significant $[F(1,14)=1.99]$.

The results of this experiment confirm those of Experiments 1 and 2 . Initial training in which pairs of stimuli ( $A$ and $C ; B$ and $D$ ) receive similar treatment enhances generalization between members of a pair so that subsequent training, establishing a high rate of responding to $C$ and a low rate to $D$, results in a higher rate of responding to $A$ than to $B$ on the test. This effect holds both when the members of a pair are accompanied by a common consequence in initial training and when they are accompanied by a common antecedent. There is some suggestion from the results of this experiment (as from those of Experiments 1 and 2) that the magnitude of the effect is greater in subjects given the backward training procedure than in those given the forward training procedure, the effect produced by the latter being particularly short-lived. But interpretation of any such difference is complicated by the (nonsignificantly) higher general response rate shown by Group Backward. Furthermore, statistical analysis does not entirely confirm the reliability of the effect and so allows only the conclusion that, for both forward and backward groups, the rate of response to $A$ early in the test is higher than that to $B$.

\section{GENERAL DISCUSSION}

Acquired equivalence has usually been established by training in which the cues in question are associated (as 
in Experiment 1 and in Group Forward of Experiment 3) with a common consequence. Our second experiment shows that training in which the cues are associated with a common antecedent can also establish equivalence, a result confirmed by the performance of Group Backward in Experiment 3. The issue we now address is whether an explanation can be devised that will accommodate both versions of the phenomenon.

The explanation we have offered for the standard (forward) case of acquired equivalence suggests that the common outcome ( $X$ in our experiments) comes to mediate generalization between the target cues $(A$ and $C$ ). Associations formed during equivalence training will allow both $A$ and $C$ to activate a representation of $X$, and this representation would then constitute an added common element that could enhance the degree of generalization between $A$ and $C$. This analysis relies on the assumption that the excitatory associations $A-X$ and $C-X$ are formed during training. In the backward equivalence procedure, however, the common event $X$ is presented before $A$ and before $C$, an arrangement that is usually assumed to be inappropriate for the formation of excitatory $A-X$ and $C-X$ associations.

An explanation for the backward equivalence effect becomes available, then, if we reject the usual assumption and allow that the backward pairings are capable, to some extent, of endowing $A$ and $C$ with the ability to activate the representation of $X$. Viewed in this way, the backward equivalence effect can be seen as providing one more piece of evidence in favor of the reality of excitatory backward conditioning to be added to those that have recently begun to emerge from studies of discrimination learning in pigeons (e.g., Richards, 1988; Zentall, Sherburne, \& Steirn, 1992).

Our results cannot be taken as decisive evidence for backward conditioning, however, since it may well be possible to devise explanations for the outcome of Experiment 2 and of Group Backward of Experiment 3 that assume only forward conditioning. Among the possibilities (see Honey \& Hall, 1991, for a discussion of others) is the following. Forward conditioning principles imply that during the first stage of training in the backward procedure, $X$ will acquire the ability to excite the representations of both $A$ and $C$. As a consequence, the $A$ representation will be active on trials when $C$ follows $X$ and the $C$ representation will be active on trials when $A$ follows $X$. If we allow that an associatively activated representation can enter into further associations (see, e.g., Holland, 1981, 1990), then $A-C$ and $C-A$ associations will be formed. These links would then generate equivalence between $A$ and $C$, since training given to one would necessarily influence the (associatively activated) representation of the other. Reinforcement of $C$, for instance, would increase the associative strength of the $A$ representation, allowing $A$ itself to evoke conditioned responding on the subsequent test. It is conceivable that chains of association of this sort might be responsible for the effects seen in other procedures that have previously been interpreted in terms of excitatory backward conditioning. But whether the assumptions involved in this sort of analysis are any more plausible than those implied by the notion of backward conditioning itself is clearly open to debate.

Finally, it is appropriate to comment on the relationship between the equivalence effects dealt with here and the work pioneered by Sidman and his colleagues on the formation of equivalence classes (e.g., Sidman \& Tailby, 1982). The type of stimulus substitutability demonstrated in our experiments is referred to by Sidman (e.g., 1990) as "functional" equivalence and is distinguished from the more formal notion of equivalence that is his central concern-Sidman adopts the logical or mathematical notion of equivalence and urges the use of behavioral tests that might be able to reveal that a relationship between events shows the properties of reflexivity, symmetry, and transitivity. Clearly it is possible (as our analysis of the studies by Urcuioli and his coworkers was intended to show) that the associative processes that might underlie the equivalence effects seen in our experiments would also be operating in the symbolic matching tasks that have frequently been employed in studies of the formation of equivalence classes. It is difficult to see, however, that these processes could generate all the effects obtained by Sidman and others. It remains possible, as Sidman (1990) has argued, that his form of the equivalence phenomenon is a "fundamental stimulus function" (Sidman, 1990, p. 111) that is not to be reduced to the operation of other processes.

\section{REFERENCES}

HALl, G. (1991). Perceptual and associative learning. Oxford: Oxford University Press, Clarendon Press.

HollaNd, P. C. (1981). Acquisition of representation-mediated conditioned food aversions. Learning \& Motivation, 12, 1-18.

Holland, P. C. (1990). Event representation in Pavlovian conditioning: Image and action. Cognition, 37, 105-131.

Honey, R. C., \& HALL, G. (1989). The acquired equivalence and distinctiveness of cues. Journal of Experimental Psychology: Animal Behavior Processes, 15, 338-346.

Honey, R. C., HaLL, G. (1991). Acquired equivalence and distinctiveness of cues using a sensory-preconditioning procedure. Quarterly Journal of Experimental Psychology, 43B, 121-135.

HuLL, C. L. (1939). The problem of stimulus equivalence in behavior theory. Psychological Review, 46, 9-30.

MILLER, N. E. (1948). Theory and experiment relating psychoanalytic displacements to stimulus-response generalization. Journal of Abnomal \& Social Psychology, 43, 155-178.

Miller, N. E., \& Dollard, J. (1941). Social learning and imitation. New Haven, CT: Yale University Press.

RichaRDS, R. W. (1988). The question of bidirectional associations in pigeons' learning of conditional discrimination tasks. Bulletin of the Psychonomic Society, 26, 577-579.

Sidman, M. (1990). Equivalence relations: Where do they come from? In D. E. Blackman \& H. Lejeune (Eds.), Behaviour analysis in the ory and practice: Contributions and controversies (pp. 93-114). Hove and London: Erlbaum. 
Sidman, M., \& TAILbY, W. (1982). Conditional discrimination vs. matching to sample: An expansion of the testing paradigm. Journal of the Experimental Analysis of Behavior, 37, 5-22.

Urculoli, P. J., \& Zentall, T. R. (in press). A test of comparisonstimulus substitutability following one-to-many matching by pigeons. Psychological Record.

Urcuiol, P. J., Zentall, T. R., Jackson-Smith, P., \& Steirn, J. N. (1989). Evidence for common coding in many-to-one matching: Retention, intertrial interference, and transfer. Joumal of Experimental Psychology: Animal Behavior Processes, 15, 264-273.

Zentall, T. R., Sherburne, L. M, STeirn, J. N. (1992). Develop- ment of excitatory backward associations during the establishment of forward associations in a delayed conditional discrimination by pigeons. Animal Learning \& Behavior, 20, 199-206.

Zentall, T. R., Steirn, J. N., Sherburne, L. M., \& Urcuioli, P. J. (1991). Common coding in pigeons assessed through partial versus total reversals of many-to-one conditional and simple discriminations. Journal of Experimental Psychology: Animal Behavior Processes, 17, 194-201.

(Manuscript received October 6, 1992; revision accepted for publication March 16, 1993.) 